\title{
CAD/CAM milled complete removable dental prostheses: An in vitro evaluation of biocompatibility, mechanical properties, and surface roughness
}

\author{
Murali SRINIVASAN ${ }^{1}$, Harald GJENGEDAL ${ }^{2}$, Maria CATTANI-LORENTE ${ }^{3}$, Mira MOUSSA ${ }^{3}$, Stéphane DURUAL ${ }^{3}$, \\ Martin SCHIMMEL ${ }^{1,4}$ and Frauke MÜLLER ${ }^{1,5}$ \\ ${ }^{1}$ Division of Gerodontology and Removable Prosthodontics, University Clinics of Dental Medicine, University of Geneva, Geneva, Switzerland \\ ${ }^{2}$ Department of Clinical Dentistry, University of Bergen, Bergen, Norway \\ ${ }^{3}$ Department of Fixed Prosthodontics and Biomaterials, University Clinics of Dental Medicine, University of Geneva, Geneva, Switzerland \\ ${ }^{4}$ Division of Gerodontology, School of Dental Medicine, University of Bern, Bern, Switzerland \\ ${ }^{5}$ Service of Geriatrics, Department of Internal Medicine, Rehabilitation and Geriatrics, University Hospitals of Geneva, Thônex, Switzerland \\ Corresponding author, Murali SRINIVASAN; E-mail: murali.srinivasan@unige.ch
}

\begin{abstract}
This study compared the biocompatibility, mechanical properties, and surface roughness of a pre-polymerized polymethyl methacrylate (PMMA) resin for CAD/CAM complete removable dental prostheses (CRDPs) and a traditional heat-polymerized PMMA resin. Two groups of resin substrates [Control (RC): conventional PMMA; Test (RA): CAD/CAM PMMA] were fabricated. Human primary osteoblasts and mouse embryonic-fibroblasts were cultured for biocompatibility assays. Mechanical properties and surface roughness were compared. ANOVA revealed no difference between the resin groups in the biocompatibility assays. RA demonstrated a higher elastic modulus ( $p=0.002)$, young's modulus $(p=0.002)$, plastic energy $(p=0.002)$, ultimate strength $(p=0.0004)$, yield point $(p=0.016)$, strain at yield point $(p=0.037)$, and toughness $(p<0.0001)$; while RC displayed a higher elastic energy $(p<0.0001)$. Laser profilometry concluded a rougher surface profile $(p<0.0001)$ for RA. This study concluded that the tested CAD/CAM resin was equally biocompatible and presented with improved mechanical properties than the traditional heat-polymerized PMMA resin used in the fabrication of CRDPs.
\end{abstract}

Keywords: CAD/CAM milled complete removable dental prostheses, Complete dentures, CAD/CAM, Laser profilometry

\section{INTRODUCTION}

The manufacturing of complete removable dental prostheses (CRDPs) by the computer-aided designing and manufacturing (CAD/CAM) methods, first documented in the $1990 \mathrm{~s}^{1,2)}$, have substantially increased in the last decade ${ }^{3}$. This technology-driven manufacturing process owes its exponential growth to a few important factors such as, but not entirely restricted to, the changing attitudes and/or trends of the clinicians as well as the dental technicians, the potential reduction in clinical chairside time and dental laboratory costs, as well as the incorporation of pre-polymerized materials. Clinical success, in terms of patient satisfaction and patient preference for the CAD/CAM manufactured CRDPs, has been reported in literature ${ }^{4-6)}$. In terms of the trueness of the CAD/CAM fabricated CRDPs, when compared to conventional CRDPs, was found to be non-inferior to a conventional PMMA and within the clinically acceptable range $\mathrm{e}^{7-10)}$.

The conventional 'flask-pack-press' technique for the fabrication of CRDPs has been employed for more than half-a-century and can be considered as a time-tested "gold standard" procedure. Traditionally, the CRDPs are manufactured with polymethyl methacrylate (PMMA) resin and involves a heat polymerization process ${ }^{11}$. Over the years, this procedure has witnessed modifications

Color figures can be viewed in the online issue, which is available at J-STAGE.

Received Jun 23, 2017: Accepted Sep 29, 2017

doi:10.4012/dmj.2017-207 JOI JST.JSTAGE/dmj/2017-207 with improvements in the PMMA resin properties and also the related processing protocol such as the use of auto-polymerizing, microwave-processing, or injectionmolding techniques ${ }^{12,13)}$. The traditional concept per se has relatively remained the same i.e., uncured PMMA resin being molded into the desired form under pressure and polymerized. However, this protocol has seen a phenomenal transition since the ingress of CAD/CAM manufacturing procedures of CRDPs.

The two common CAD/CAM techniques used in the manufacturing of CRDPs, rapid prototyping and CAD/ CAM milling techniques, employ protocols that are totally contrasting to the traditional techniques. The rapid prototyping or additive $\mathrm{CAD} / \mathrm{CAM}$ process involves a serial apposition of the resin material on a support structure followed by curing/hardening by visible light, UV light, heat or laser. This accumulative layering and curing process is repeated until the CAD-designed CRDP form is achieved. The subtractive or CAD/CAM milling process, on the other hand, involves the CRDP to be milled out from a pre-polymerized commerciallymanufactured PMMA blank (puck). This blank is produced under high pressure and is therefore claimed to have superior mechanical properties, exhibit better hygienic properties, and leech out less residual monomer. However, no significant differences could be established in the amount of monomer leeched out from CAD/ CAM milled CRDPs and conventionally manufactured $\mathrm{CRDP}^{14)}$. The other assumptions however, have not yet 
been substantiated in literature.

Although these resins are chemically similar to the traditional PMMA resins used in the fabrication of conventional CRDPs, fact still remains that their production process is entirely different. Whether the PMMA resins manufactured by newer protocols are able to retain their original mechanical properties and function under clinical conditions successfully over a long-term, need to be scientifically warranted. Moreover, independent evidence relating to the biocompatibility and mechanical properties of these PMMA resins employed for the milling of the CRDPs are non-existent in scientific literature.

Hence, this study was conducted with the aim of evaluating the biocompatibility assays, mechanical properties, and the surface roughness of one type of pre-polymerized PMMA resin that is used in the manufacturing of CAD/CAM milled CRDPs. The null hypothesis which was set for this in vitro study was that there is no difference in the biocompatibility, mechanical properties, and surface roughness between the prepolymerized PMMA resin used in the manufacturing of CAD/CAM milled CRDPs and the traditional PMMA resin used in conventional heat polymerized CRDPs.

\section{MATERIALS AND METHODS}

\section{Resin specimens}

The control samples used in this study were custommade from conventional heat polymerized PMMA resin. The test specimens were milled from a pre-polymerized PMMA resin puck.

\section{Control group (RC)}

Resin specimens were custom-manufactured from conventional heat polymerized PMMA resin (Aesthetic Red, CANDULOR, Glattpark, Switzerland) in a conventional dental laboratory by a master dental technician. Wax-patterns of the required dimensions of the specimens were cut and then processed to PMMA by the conventional flask, pack and press fabrication method. After polymerization, the specimens were trimmed for excess, finished and polished using pumice powder (Polyglass Ultra ponce, KALADENT, Urdorf, Switzerland) and felt-tip polishing buffs. Final finishing was done using a universal high-gloss polishing paste intended for PMMA resins (KMG, CANDULOR, Wangen, Switzerland) with a high luster polishing buff (High luster buff Acryl, Bredent, Senden, Germany).

\section{Test group (RA)}

A pre-polymerized PMMA puck, manufactured under high pressure (AVADENT, Global Dental Science Europe, Tilburg, The Netherlands), was used for manufacturing the resin specimens for this group. The specimens were cut using a rotary table saw (Inca, Injecta, Teufenthal, Switzerland) equipped with a 3 $\mathrm{mm}$ thick stainless circular blade (Oertli Werkzeuge, Höri, Switzerland). The cut resin specimens were then finished and polished by the master dental technician.
The finishing and polishing procedures followed were the same as described for control group. The cut specimens were stored in a sterile plastic container in a cool dry place before testing.

3. Number and dimensions of specimens

A total of 30 resin specimens per group were fabricated with specific dimensions for the various tests conducted, as enumerated below:

1) Biocompatibility assays $(n=18)$ : dimensions: $11 \times 11 \times 2 \mathrm{~mm}$

2) Mechanical properties testing:

a. Nanoindentation test $(n=2)$ : dimensions: $11 \times 11 \times 2 \mathrm{~mm}$

b. Three-point bending test $(n=5)$ : dimensions: $65 \times 10 \times 3 \mathrm{~mm}$

c. Surface roughness test $(n=5)$ : dimensions: $20 \times 20 \times 1.5 \mathrm{~mm}$

4. Disinfection of specimen

After final polishing, the resin substrates were disinfected for culture and mechanical testing. The specimens were placed in $70 \%$ ethanol solution for 5 min, and subsequently dried with sterile cotton. They were sterilized for $60 \mathrm{~min}$ under ultra-violet light with a wavelength of $254 \mathrm{~nm}$ emitted by a 15 watt UV lamp present in the safety cabinet (SafeFAST premium 212, LogicAir, St-Aubin, Switzerland).

\section{Biocompatibility assays}

1. Cells

Two types of cells were cultured on the substrates separately,

1) Human primary osteoblasts (hOBs),

2) Embryological mouse fibroblasts (3T3 cells, ATCC CRL-1658)

Frequently used human primary osteoblasts cell line, cultured in our laboratory of biomaterials was used for the assays ${ }^{15}$. For the analysis, passages $3-5$ were used as described in previous similar experiments ${ }^{15,16)}$. The fibroblast cell line (3T3 cells) used in this experiment is a well-established standard cell line cultured from the embryonic tissue of the Swiss albino mouse.

The density of the cells used for the assays was 2,600 cells $/ \mathrm{cm}^{2}$ cultures in 24-well plates (TPP techno Plastic Products, Trasadingen, Switzerland) with Dulbecco's Modified Eagle's Medium (DMEM medium, Life Technologies, Carlsbad, CA, USA), in which $10 \%$ foetal calf serum (Eurobio, Les Ulis, France), 1\% penicillin/ streptomycin/fungizone (Life Technologies), 2\% HEPES (Life Technologies) were further supplemented. The $\mathrm{RC}$ and RA plates were immersed in this medium for incubation and culture for both cell lines.

2. Cell culture and proliferation (Resazurin assay)

The hOBs and 3T3 cells were cultured on both, RC and RA substrates plates. For each cell line (hOBs and 3T3) and each group, cells were cultured for 21 days in triplicate. This assay was repeated three times $(n=9)$ for the two cell lines separately as described in earlier 
publications ${ }^{16)}$. Resazurin assays were done on days 3 , 7, 14 and 21 within each test run. Once the cultures were ready, on the day of measurement, Resazurin (Resazurin Sodium salt, Sigma Aldrich, St. Louis, MO, USA) with a concentration of $10 \mu \mathrm{g} / \mathrm{mL}$ was added in the culture media to measure the proliferation of the cells. The cells were maintained at $37^{\circ} \mathrm{C} / 5 \% \mathrm{CO}_{2}$ for $4 \mathrm{~h}$. Resazurin is transformed to Resofurin during this incubation. The absorbance of resofurin in culture media was assessed at 570 and $630 \mathrm{~nm}$. The percentage of reduction of the resazurin was then calculated according to the manufacturer's instructions and used for data analysis.

\section{Mechanical properties and surface characteristics 1. Nanoindentation test}

A nanoindenter equipped with a Berkovich indenter (CSM Instrument, Peseux, Switzerland) was used to perform the tests. The Berkovich diamond tip was calibrated using a fused silica standard provided by the manufacturer. A load of $8 \mathrm{mN}$ was applied at a rate of 76 $\mathrm{mN} \cdot \mathrm{min}^{-1}$. At maximum constant load, a 10-s holding period was imposed. The applied load and penetration depth were continuously recorded during the loading and unloading cycle. Five indentations were placed for each specimen $(n=2)$ at different random locations. Elastic modulus and hardness were obtained from the unloading portion of the indentation curves, using the Oliver and Pharr method ${ }^{17)}$. The elastic and plastic energies needed to perform the indents were also estimated. To compute the elastic modulus, Poisson's ration was taken as 0.3.

\section{Three-point bending test}

A three-point bending test was performed to further assess the mechanical properties of the resins. Five samples were stored in water at $37^{\circ} \mathrm{C}$ for $24 \mathrm{~h}$. The span length of the specimen was $50 \mathrm{~mm}$ and a vertical load was applied at the mid-point of the specimen at a crosshead speed of $1 \mathrm{~mm} / \mathrm{min}$ by a universal testing machine (INSTRON 1117, INSTRON, Norwood, MA, USA). The ultimate strength, flexural elastic modulus, stress at the proportional limit (yield point), flexural strain at the proportional limit, and toughness were determined for each sample.

\section{Laser profilometry}

Five samples per group were measured and the corresponding profiles were generated per sample. Surface roughness profile (R) was measured using a high-resolution white light non-contact laser profilometer (CyberSCAN CT 100, Cyber technologies, Eching-Dietersheim, Germany) with a z-resolution of $20 \mathrm{~nm}$ and a lateral resolution of $1 \mu \mathrm{m}$; $\mathrm{R}$ was calculated using a Gaussian profile filter with the cut-off wavelength $\left(\lambda_{c}\right)$ set to $0.8 \mathrm{~mm}$ and the sampling length to $4 \mathrm{~mm}$, in a given total scanning length of $5.6 \mathrm{~mm}$ as per the specifications by the International Standards Organization (ISO 11562). This total scanning length is then split into five sampling lengths. $\mathrm{R}$ is analyzed within this total scanning length, and the most commonly measured roughness characteristics, average roughness $\left(R_{a}\right)$ and maximum roughness $\left(R_{\max }\right)$ were analyzed. $R_{a}$ is the arithmetic mean value of all heights (peaks and valleys) in the given roughness profile. $R_{\max }$ is the maximum of all roughness depths (distance between the deepest valley and the highest peak) measured within the complete scan length.

\section{Statistical analysis}

Data collected were verified for normal distribution using the Kolmogorov-Smirnov test and compared for statistical significance using the student's $t$-tests and two-way ANOVA. The level of statistical significances for all tests were set at $p<0.05$. All statistical analyses were done using Statistica ver.12 (Dell, Tulsa, OK, USA), software package.

\section{RESULTS}

Biocompatibility assays (hOBs and 3T3 cells proliferation) The hOBs and 3T3 cells' activities in the RC group at day 3 were set as baseline. Cells proliferated regularly and gradually on both surfaces, showing a 10-fold increase from day 3 to day 21 for the osteoblasts, and a 20 -fold increase for the fibroblasts. The ANOVA model revealed no significant differences between the two groups for either the hOBs $[\mathrm{F}(1,64)=1.177, p=0.283]$ or 3 T3 cells $[\mathrm{F}(1,64)=0.186, p=0.668]$ proliferation (Table 1$)$.

\section{Mechanical properties and surface characteristics}

1. Nanoindentation test

RA group displayed a significantly higher elastic modulus ( $p=0.002)$ and the young's modulus $(p=0.002)$ than the RC group (Table 2). Although the total energy needed to perform the indents on the resin substrates was the same for both groups, their plastic and elastic behaviors differed; RA displayed a higher plastic energy $(p=0.002)$ but a lower elastic energy than $\mathrm{RC}$ $(p<0.0001)$. The hardness of RA and RC were not statistically significant.

\section{Three-point bending test}

During exposure to continuously increasing load, all five samples fractured in the RC group but only two samples fractured in the RA group. The three non-fractured samples in the RA group, displayed a large deflection when the experiment had reached its force limits. These non-fractured RA samples returned to what seemed their original shape in some time after the applied load had been removed. Although both resins showed similar flexural elastic modulus $(p=0.712)$, RA group was superior in its ultimate strength $(p=0.0004)$, yield point $(p=0.016)$, strain at yield point $(p=0.037)$, and toughness $(p<0.0001)$ when compared to the RC group (Table 3$)$.

\section{Laser profilometry}

The profilometry results revealed that both the $R_{a}$ and the $R_{\max }$ were found to be significantly higher in the RA group when compared to the RC group $(p<0.0001$; Table 4, Fig. 1). 
Table 1 Results of the two-way ANOVA model for the percentage (\%) of fibroblast (3T3 cells) and osteoblast (hOBs) growth on the two resin substrates observed at different time points; with the confidence intervals set at $95 \%$ and level of significance set to $p<0.05$

\begin{tabular}{|c|c|c|c|c|c|c|c|c|}
\hline \multirow{2}{*}{ Group } & \multicolumn{4}{|c|}{ 3T3 cells growth (mean \pm standard deviation) } & \multicolumn{4}{|c|}{ hOBs growth (mean \pm standard deviation) } \\
\hline & Day 3 & Day 7 & Day 14 & Day 21 & Day 3 & Day 7 & Day 14 & Day 21 \\
\hline $\mathrm{RC}$ & $1.113 \pm 0.40$ & $6.272 \pm 0.81$ & $23.998 \pm 7.82$ & $20.852 \pm 5.42$ & $1.259 \pm 0.34$ & $4.176 \pm 1.79$ & $11.113 \pm 5.93$ & $10.936 \pm 5.71$ \\
\hline RA & $1.092 \pm 0.24$ & $6.333 \pm 1.40$ & $25.643 \pm 8.79$ & $25.701 \pm 7.71$ & $2.260 \pm 0.71$ & $7.700 \pm 3.23$ & $17.101 \pm 7.97$ & $15.836 \pm 7.51$ \\
\hline \multicolumn{9}{|c|}{ Two-way ANOVA results } \\
\hline $\begin{array}{c}\text { Biocompatibility } \\
\text { test }\end{array}$ & \multicolumn{2}{|c|}{$\begin{array}{l}\text { Source of } \\
\text { variation }\end{array}$} & SS & df & \multicolumn{2}{|l|}{ MS } & $\mathrm{F}$ & $p$-value \\
\hline \multirow{5}{*}{ 3T3 cells } & \multicolumn{2}{|c|}{ Groups } & 48.021 & 1 & \multicolumn{2}{|c|}{48.021} & 0.186 & 0.668 \\
\hline & \multirow{2}{*}{\multicolumn{2}{|c|}{ Days }} & $7,715.678$ & 3 & \multicolumn{2}{|c|}{$2,571.893$} & 9.945 & $<0.0001$ \\
\hline & & & 69.993 & 3 & \multicolumn{2}{|c|}{23.331} & 0.090 & 0.965 \\
\hline & \multicolumn{2}{|c|}{$\begin{array}{l}\text { Interaction } \\
\text { Residual }\end{array}$} & $16,551.070$ & 64 & \multirow{2}{*}{\multicolumn{2}{|c|}{258.610}} & & \\
\hline & \multicolumn{2}{|c|}{ Total } & $24,384.762$ & 71 & & & & \\
\hline \multirow{5}{*}{ hOBs } & \multicolumn{2}{|c|}{ Groups } & 267.280 & 1 & \multicolumn{2}{|c|}{267.280} & 1.177 & 0.283 \\
\hline & \multicolumn{2}{|c|}{ Days } & $1,925.145$ & 3 & \multicolumn{2}{|c|}{641.715} & 2.825 & 0.046 \\
\hline & \multicolumn{2}{|c|}{ Interaction } & 62.540 & 3 & \multirow{2}{*}{\multicolumn{2}{|c|}{$\begin{array}{r}20.847 \\
227.157\end{array}$}} & 0.092 & 0.964 \\
\hline & \multicolumn{2}{|c|}{ Residual } & $14,538.047$ & 64 & & & & \\
\hline & \multicolumn{2}{|c|}{ Total } & $16,793.012$ & 71 & \multicolumn{2}{|c|}{227.157} & & \\
\hline
\end{tabular}

Table 2 Nanoindentation test results for the control (RC) and test (RA) groups

\begin{tabular}{lccccc}
\hline Group & $\begin{array}{c}\text { Elastic modulus } \\
(\mathrm{GPa})\end{array}$ & $\begin{array}{c}\text { Young's modulus } \\
(\mathrm{GPa})\end{array}$ & $\begin{array}{c}\text { Hardness } \\
(\mathrm{MPa})\end{array}$ & $\begin{array}{c}\mathrm{W}_{\text {elast }} \\
(\mathrm{mN} . \mathrm{mm})\end{array}$ & $\begin{array}{c}\mathrm{W}_{\text {plast }} \\
(\mathrm{mN} . \mathrm{mm})\end{array}$ \\
\hline RC & $3.9 \pm 0.2$ & $4.2 \pm 0.2$ & $232 \pm 15$ & $1,481 \pm 31$ & $3,469 \pm 82$ \\
RA & $4.1 \pm 0.2$ & $4.5 \pm 0.2$ & $221 \pm 14$ & $1,319 \pm 18$ & $3,727 \pm 204$ \\
$p$-value & 0.002 & 0.002 & 0.091 & $<0.0001$ & 0.002 \\
\hline
\end{tabular}

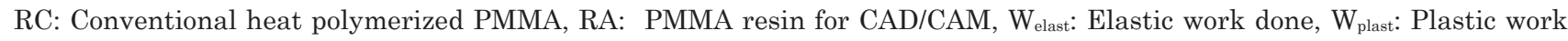
done, $\mathrm{RC}$ versus $\mathrm{RA}$

Table 3 Three-point bending test results for the control (RC) and test (RA) groups

\begin{tabular}{lccccc}
\hline Group & $\begin{array}{c}\text { Ultimate strength } \\
(\mathrm{GPa})\end{array}$ & $\begin{array}{c}\text { Flexural elastic } \\
\text { modulus }(\mathrm{GPa})\end{array}$ & $\begin{array}{c}\text { Yield point } \\
(\mathrm{MPa})\end{array}$ & $\begin{array}{c}\text { Strain at } \\
\text { yield point }\end{array}$ & $\begin{array}{c}\text { Toughness } \\
(\mathrm{N} . \mathrm{mm})\end{array}$ \\
\hline RC & $96 \pm 4$ & $2.7 \pm 0.1$ & $54 \pm 11$ & $0.020 \pm 0.005$ & $436 \pm 46$ \\
RA & $121 \pm 2$ & $2.7 \pm 0.2$ & $71 \pm 6$ & $0.003 \pm 0.002$ & $956 \pm 85$ \\
$p$-value & 0.0004 & 0.712 & 0.016 & 0.037 & $<0.0001$ \\
\hline
\end{tabular}

RC: Conventional heat polymerized PMMA, RA: PMMA resin for CAD/CAM

Table 4 Comparison of the mean of all heights in the roughness profile $\left(R_{a}\right)$ and the maximum height of the roughness profiles $\left(\mathrm{R}_{\max }\right)$ for the control (RC) and test (RA) groups

\begin{tabular}{lcc}
\hline Group & $\mathrm{R}_{\mathrm{a}}(\mathrm{in} \mu \mathrm{m})^{*}$ & $\mathrm{R}_{\max }(\mathrm{in} \mu \mathrm{m}) *$ \\
\hline RC & $0.12 \pm 0.29$ & $0.92 \pm 0.37$ \\
RA & $0.37 \pm 0.03$ & $2.38 \pm 0.26$ \\
$p$-value & $<0.0001$ & $<0.0001$ \\
\hline
\end{tabular}

\footnotetext{
*: Mean \pm standard deviation, RC: Conventional heat polymerized PMMA, RA: PMMA resin for CAD/CAM
} 


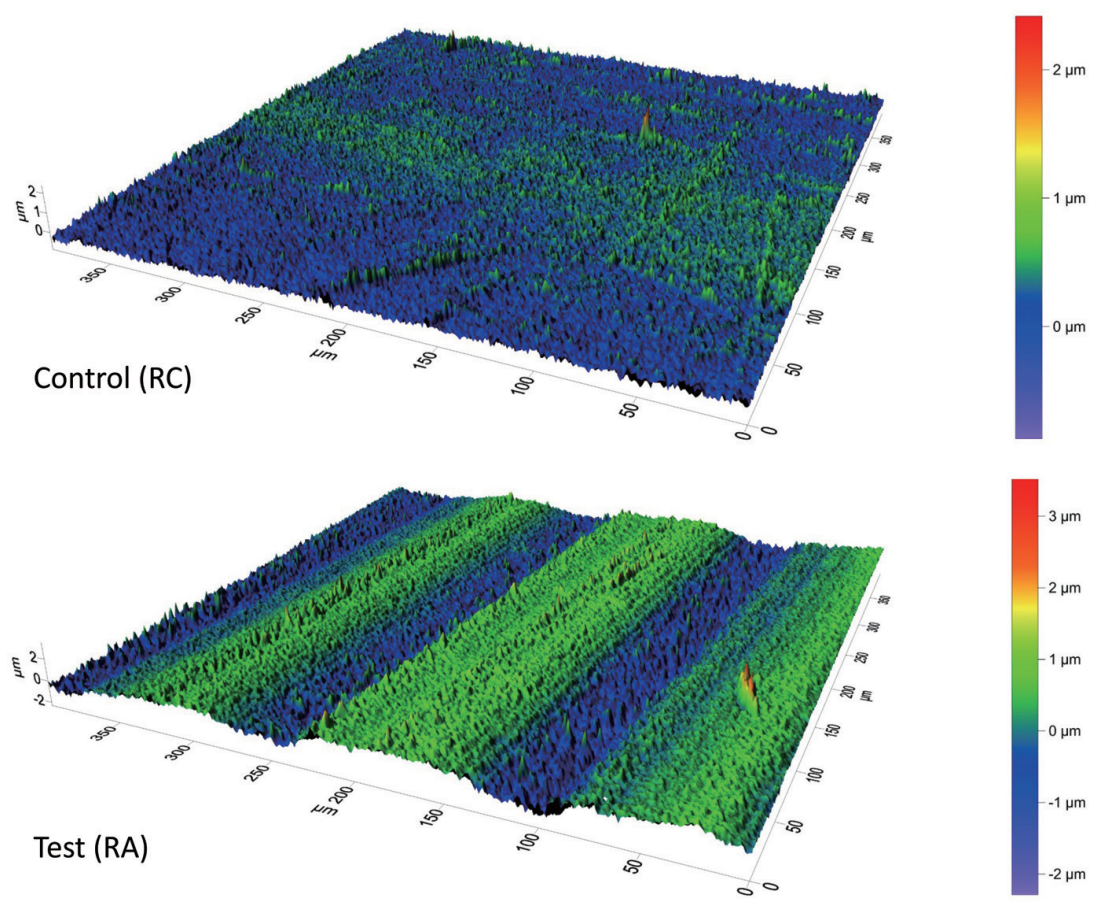

Fig. 1 Typical profilometer scan images of a test specimen from control (RC) and test (RA) groups.

\section{DISCUSSION}

The fabrication of CRDPs by CAD/CAM milling technique involves a subtractive process where a prepolymerized PMMA blank puck is milled into the designed denture form. This method of fabrication is entirely contrary to the traditional method, wherein the uncured resin is molded into form under pressure and then polymerized. The conventional method of heat polymerization of CRDPs has been in existence for many decades and has proven to be a reliable method, but has its drawbacks. Common heat polymerization errors are frequently encountered in conventional denture processing and include denture porosity, crazing, denture warpage including volumetric and linear shrinkages ${ }^{18,19}$. Denture porosity, which may be internal, external or ubiquitous, is usually caused due to the evaporation of the monomer caused by uncontrolled high temperatures during the curing cycle, or can be caused by a non-homogenous mixing of the resin and/or an inadequate pressure during its packing. Crazing, on the other hand, is the formation of cracks on the resin surface which may be stress induced, or the incorporation of water during polymerization and/or because of a solvent attack. Warpage can occur due to stresses incorporated during polymerization ${ }^{20-22}$. Volumetric and linear shrinkages of the resin during the polymerization process is inherent to the conventional flask-pack-press technique, requiring occlusal adjustments immediately after de-flasking and carving of a post-dam to compensate for a potential lift-off of the palatal plate from the plaster model. The shrinkage is reported to be 0.42 to $0.58 \%$ at water saturation which is within a clinically acceptable range $^{19)}$. The CAD/CAM milling process supposedly eliminates the above-mentioned processing errors, owing to the use of a pre-polymerized puck, which is manufactured under high pressure. The resin is said to also exhibit improved mechanical properties, better biocompatibility, surface characteristics and excellent bio-hygienic properties. However, all these claims have not been tested independently. Reports on the evaluation of the precision of the CAD/CAM manufacturing process $^{7,8)}$, and on the residual monomer content exist in current literature ${ }^{14)}$. In a study by Steinmassl et al. ${ }^{14)}$, no significant difference was observed between the monomer content leeched out from different $\mathrm{CAD} /$ CAM milled CRDPs and the conventionally fabricated dentures. In terms of precision of the fabricated methods $\mathrm{CAD} / \mathrm{CAM}$, studies have verified that the trueness of the milling methods are either better than conventional methods or remain within clinically acceptable limits ${ }^{7,8)}$. This bench experiment, therefore, compared the biocompatibility, mechanical properties, and surface characteristics of the CAD/CAM CRDP resin with a conventional PMMA resin used in the fabrication of conventional CRDPs, by testing our null hypothesis that there is no difference in the biocompatibility, mechanical properties, and surface roughness between the prepolymerized PMMA resin used in the manufacturing of CAD/CAM milled CRDPs and the traditional PMMA resin used in conventional heat polymerized CRDPs.

Inherent shortcomings are due to the chosen 
methodology in these assays and mechanical bench experiments. Resin substrates were custom prepared. The specimens for the RC group was manufactured by conventional methods. A wax pattern in the required dimension was first made, then this wax pattern was processed in a conventional PMMA resin and heat polymerized using the traditional flask-pack-press procedure. The polymerized specimens were trimmed, finished and polished by a master dental technician. While, the specimens for the RA group were milled out from the blanks and then hand finished by the same master dental technician. This methodology was typically followed to ensure that standardization and originality of the manufacturing techniques are maintained, but differences may, or may not have occurred since cutting of the pre-polymerized specimen may have introduced an additional surface roughness, as well as internal tensions in the resin, which may not have been present in the conventional specimen. Another source of surface degradation which may have occurred is while the specimens were being disinfected using ethanol solution. This however, can be argued that the exposure to ethanol during this disinfection process was a relatively short period (5 min). Moreover, both groups underwent identical protocols of disinfection and also, the chemical component (PMMA) of the resins were similar, it may be assumed that whatever negligible surface change which might have occurred in one resin group would have been consistent in both resin groups. However, it is quite possible that one resin group may have undergone more surface degradation than the other, but this was not investigated in the current experiment as it was beyond the scope of this study.

Biocompatibility of dental materials used for dental prostheses is one of highest priorities in a clinical setting. Adverse reactions of the oral mucosa, in close contact with the foreign material introduced may cause pain, hypersensitivity reactions as well as burning mouth sensations ${ }^{23,24)}$. Hence patient safety must be assured using biocompatibility testing. The cell lines (hOBs and the 3T3 cells) cultured for the biocompatibility assays are standard well established cell lines commonly used for the biocompatibility evaluation of materials in our laboratory ${ }^{15,16,25)}$. Biocompatibility assays revealed relatively good cell proliferations of both types of cells cultured, hOBs as well as the $3 \mathrm{~T} 3$ cells, on both the resin substrates. Although in a clinical situation the resins will almost never be in a direct contact with osteoblasts, it was still considered worthwhile to perform this test as a measure of biocompatibility. The results were interesting as a gradual and healthy hOBs proliferation on both resin substrates were noticed. Although there seemed visibly a higher proliferation of the hOBs on the test group (RA), a statistical significance could not be established. The 3T3 cell assay revealed a similar trend as the hOBs assay for the test group (RA), but again not statistically significant. Hence, based on the results of our present experiment, the null hypothesis with regards to the biocompatibility was not rejected as there was no difference between the two groups.
These preliminary assays sufficiently validate the noninferiority of the test group (RA) against the control. However, in a previous experiment, we noticed a significantly higher variability of the trueness of the milled CAD/CAD intaglio surfaces ${ }^{8}$. Such "terracing" may be related to the size of the milling instruments, as could be interpreted from previously published images ${ }^{7)}$. The present experiments also confirm a higher roughness of the $\mathrm{CAD} / \mathrm{CAM}$ resin specimen, hence the null hypothesis with regards to surface roughness was rejected. We noticed a tendency to an increased proliferation of both cell lines on the test group substrates. The assumed lower porosity of the puck as well as its established increased surface roughness might have fostered the cell proliferation. On the other hand, an increased roughness may foster biofilm adhesion in vivo. Surface roughness, amongst other factors like material properties, is a known contributor to oral biofilm adhesion ${ }^{26,27)}$. Hence the absence of a significant difference in cell growth may be due to two counter-acting effects. On the one side, a positive effect from a denser structure of the pre-polymerized test-resin, which fosters biocompatibility. On the other hand, a negative effect of biocompatibility might counteract this effect due to a higher surface roughness. Accumulation of oral biofilm is a highly relevant risk factor in geriatric populations ${ }^{28)}$, where the presence of oral bacteria may cause aspiration pneumonia ${ }^{29)}$, especially when the dentures are worn during the night ${ }^{30}$. At this stage, a better or worse biocompatibility of the novel prepolymerized denture resins remains speculative. No bench experiment can replace clinical trials to verify biocompatibility and biofilm adhesion in a clinical context. Hence, further biocompatibility tests to evaluate the toxicity of the resin, along with the resistance to candida and other microbial plaque adhesions due to the increased surface roughness, could prove interesting in order to provide a deeper insight.

The present study reveals mechanical properties that are distinctly different from the conventional heatpolymerized denture resin, hence the null hypothesis with regards to mechanical properties is rejected. The increased toughness and ultimate strength along with a higher elastic modulus of the RA may allow for denture designs with lower minimal thicknesses, without risking frequent denture repair. Although large parts of a dental prosthesis replace lost tissues, like teeth and alveolar bone, there are some parts where the removable denture adds additional volume to the oral cavity, notably on the hard palate. Covering the hard palate is necessary for achieving physical denture retention and for distributing occlusal load to a large surface of denture bearing tissues, but the central palate is not subject to atrophy after tooth extraction. The novel denture resins would allow a thinner palatal plate without compromising the mechanical properties of the prosthesis, hence providing the patient with less additional volume ${ }^{31}$. Advantages of a thinner palate may comprise a more natural speech, a more natural "feel" and a higher comfort, although evidence is for the moment lacking. Our first clinical 
experience, however, confirm an excellent patient compliance concerning the thin palatal plate.

Given that the blanks are pre-polymerized, volumetric and linear shrinkages have already taken place before shaping the final denture. Hence forming the denture is assumed to be devoid of any such distortions. Although one might assume a clinical advantage from this feature in terms of adaptation of the palatal plate to the tissues, previous experiments from our group could not confirm a superiority of the $\mathrm{CAD} / \mathrm{CAM}$ technique in terms of adaptation to the palate. All mean deviations in trueness in the three tested techniques, in our previous experiment, remained within a clinically acceptable range ${ }^{8)}$.

The tested CAD/CAM resin presents a high modulus of elasticity which means it takes longer to deform before breaking. Although this may correlate to a lower frequency of denture repair, it cannot be ignored, that this clinical advantage may be diluted by the use of a thinner palatal plate. It also has to be considered, that a denture which is resistant to deformation assures a stable occlusion and hence a solid positioning of the mandible against the cranium. Dentures fabricated from flexible materials like nylon or those relined with soft tissue conditioners are not adequately resistant to occlusal forces and have been associated with lower chewing efficiency and occlusal forces ${ }^{32}$. Given the bulk of the prosthesis rehabilitating the dental arch, a deformation in a clinical context is unlikely to occur, especially when the denture teeth are also milled from the same puck. Deformation under habitual chewing forces of the new CAD/CAM resins has yet to be further tested in a clinical context.

The mechanical properties of the novel CAD/CAM resins may also allow for overdentures without metal or fiber reinforcement, as the inherent toughness may prevent fractures and crack propagations in areas where voluminous attachment systems require substantial thinning of the denture bases. One might even go so far, as to try fixed implant supported dental prosthesis without metal or fiber reinforcement. Again, clinical proof for these novel treatment concepts is missing. Last but not the least, CAD/CAM resins may provide additional comfort to oncology patients wearing maxillofacial prostheses. Substantial weight savings may occur when the walls of a maxillo-facial obturator can be hollowed out to a thinner layer, without compromising the mechanical properties or the seal against liquids from the oral and nasal cavity. The long-term clinical performance of this $\mathrm{CAD} / \mathrm{CAM}$ resin however, needs to be further evaluated by prospective clinical trials.

\section{CONCLUSIONS}

This study revealed that the tested CAD/CAM PMMA resin is equally biocompatible and presented with improved mechanical properties than the traditional heat-polymerized PMMA resin used in the fabrication of CRDPs. With its improved mechanical properties, the $\mathrm{CAD} / \mathrm{CAM}$ resin may be suitable for a wider range of prosthetic designs specifically in special clinical indications.

\section{ACKNOWLEDGMENTS}

Jean-Charles Meinhardt, master dental technician is acknowledged for the help in preparing all the different resin samples used in this study. The authors dedicate this paper to the memory of Dr. Maria Cattani-Lorente who passed away in a road-traffic accident before finishing this manuscript.

\section{CONFLICTS OF INTEREST}

The authors report that they have no conflicts of interests related to this study. This bench study was entirely funded by institutional funds of the Division of Gerodontology and Removable Prosthodontics, University Clinics of Dental Medicine, University of Geneva, Geneva, Switzerland. The laser scanner used in this study was acquired by a generous grant (SSO Grant No. 276-13) awarded by the Swiss Dental Association (SSO-Schweizerische Zahnärzte Gesellschaft) to Professor Susanne Scherrer, Division of Fixed Prosthodontics and Biomaterials, University Clinics of Dental Medicine, University of Geneva, Geneva, Switzerland.

\section{REFERENCES}

1) Kawahata N, Ono H, Nishi Y, Hamano T, Nagaoka E. Trial of duplication procedure for complete dentures by CAD/CAM. J Oral Rehabil 1997; 24: 540-548.

2) Maeda Y, Minoura M, Tsutsumi S, Okada M, Nokubi T. A $\mathrm{CAD} / \mathrm{CAM}$ system for removable denture. Part I: Fabrication of complete dentures. Int J Prosthodont 1994; 7: 17-21.

3) Baba NZ, AlRumaih HS, Goodacre BJ, Goodacre CJ. Current techniques in CAD/CAM denture fabrication. Gen Dent 2016; 64: 23-28.

4) Bidra AS, Farrell K, Burnham D, Dhingra A, Taylor TD, Kuo CL. Prospective cohort pilot study of 2-visit CAD/ CAM monolithic complete dentures and implant-retained overdentures: Clinical and patient-centered outcomes. J Prosthet Dent 2016; 115: 578-586.

5) Kattadiyil MT, Jekki R, Goodacre CJ, Baba NZ. Comparison of treatment outcomes in digital and conventional complete removable dental prosthesis fabrications in a predoctoral setting. J Prosthet Dent 2015; 114: 818-825.

6) Pereyra NM, Marano J, Subramanian G, Quek S, Leff D, Comparison of patient satisfaction in the fabrication of conventional dentures vs. DENTCA (CAD/CAM) dentures: A case report. J N J Dent Assoc 2015; 86: 26-33.

7) Goodacre BJ, Goodacre CJ, Baba NZ, Kattadiyil MT. Comparison of denture base adaptation between CAD/CAM and conventional fabrication techniques. J Prosthet Dent 2016; 116: 249-256.

8) Srinivasan M, Cantin Y, Mehl A, Gjengedal H, Müller F, Schimmel M. CAD/CAM milled removable complete dentures: an in vitro evaluation of trueness. Clin Oral Investig 2016; 21: 2007-2019.

9) Chen H, Wang H, Lv P, Wang Y, Sun Y. Quantitative evaluation of tissue surface adaption of CAD-designed and $3 \mathrm{D}$ printed wax pattern of maxillary complete denture. Biomed Res Int 2015; 2015: 453968.

10) Inokoshi M, Kanazawa M, Minakuchi S. Evaluation of a 
complete denture trial method applying rapid prototyping. Dent Mater J 2012; 31: 40-46.

11) Matsuo H, Suenaga H, Takahashi M, Suzuki O, Sasaki K, Takahashi N. Deterioration of polymethyl methacrylate dentures in the oral cavity. Dent Mater J 2015; 34: 234-239.

12) Nogueira SS, Ogle RE, Davis EL. Comparison of accuracy between compression- and injection-molded complete dentures. J Prosthet Dent 1999; 82: 291-300.

13) Parvizi A, Lindquist T, Schneider R, Williamson D, Boyer D, Dawson DV. Comparison of the dimensional accuracy of injection-molded denture base materials to that of conventional pressure-pack acrylic resin. J Prosthodont 2004; 13: 83-89.

14) Steinmassl PA, Wiedemair V, Huck $C$, Klaunzer F, Steinmassl O, Grunert I, Dumfahrt H. Do CAD/CAM dentures really release less monomer than conventional dentures? Clin Oral Investig 2016; 21: 1697-1705.

15) Durual S, Pernet F, Rieder P, Mekki M, Cattani-Lorente M, Wiskott HW. Titanium nitride oxide coating on rough titanium stimulates the proliferation of human primary osteoblasts. Clin Oral Implants Res 2011; 22: 552-559.

16) Moussa M, Fontana P, Hamdan F, Cattani-Lorente M, Scherrer SS, Banakh O, Wiskott AH, Durual S. Modulation of osteoblast behavior on TiNxOy coatings by altering the N/O stoichiometry while maintaining a high thrombogenic potential. J Biomater Appl 2016; 30: 1219-1229.

17) Oliver WC, Pharr GM. An improved technique for determining hardness and elastic modulus using load and displacement sensing indentation experiments. J Mater Res 1992; 7: 15641583.

18) Woelfel JB, Paffenbarger GC, Sweeney WT. Dimensional changes occurring in dentures during processing. J Am Dent Assoc 1960; 61: 413-430.

19) Wong DM, Cheng LY, Chow TW, Clark RK. Effect of processing method on the dimensional accuracy and water sorption of acrylic resin dentures. J Prosthet Dent 1999; 81: 300-304.

20) Firtell DN, Green AJ, Elahi JM. Posterior peripheral seal distortion related to processing temperature. J Prosthet Dent 1981; 45: 598-601.

21) Sanders JL, Levin B, Reitz PV. Comparison of the adaptation of acrylic resin cured by microwave energy and conventional water bath. Quintessence Int 1991; 22: 181-186.

22) Sykora O, Sutow EJ. Posterior palatal seal adaptation: influence of processing technique, palate shape and immersion. J Oral Rehabil 1993; 20: 19-31.

23) Kanerva L, Jolanki R, Estlander T. 10 years of patch testing with the (meth)acrylate series. Contact Dermatitis 1997; 37: 255-258.

24) Vilaplana J, Romaguera C. Contact dermatitis and adverse oral mucous membrane reactions related to the use of dental prostheses. Contact Dermatitis 2000; 43: 183-185.

25) Durual S, Rieder P, Garavaglia G, Filieri A, Cattani-Lorente M, Scherrer SS, Wiskott HW. TiNOx coatings on roughened titanium and $\mathrm{CoCr}$ alloy accelerate early osseointegration of dental implants in minipigs. Bone 2013; 52: 230-237.

26) Jackson S, Coulthwaite L, Loewy Z, Scallan A, Verran J. Biofilm development by blastospores and hyphae of Candida albicans on abraded denture acrylic resin surfaces. J Prosthet Dent 2014; 112: 988-993.

27) Verran J, Jackson S, Coulthwaite L, Scallan A, Loewy Z, Whitehead K. The effect of dentifrice abrasion on denture topography and the subsequent retention of microorganisms on abraded surfaces. J Prosthet Dent 2014; 112: 1513-1522.

28) Baumgartner W, Schimmel M, Müller F. Oral health and dental care of elderly adults dependent on care. Swiss Dent J 2015; 125: 417-426.

29) Sjogren P, Wardh I, Zimmerman M, Almstahl A, Wikstrom M. Oral care and mortality in older adults with pneumonia in hospitals or nursing homes: Systematic review and metaanalysis. J Am Geriatr Soc 2016; 64: 2109-2115.

30) Inuma T, Arai Y, Abe Y, Takayama M, Fukumoto M, Fukui Y, Iwase T, Takebayashi T, Hirose N, Gionhaku N, Komiyama K. Denture wearing during sleep doubles the risk of pneumonia in the very elderly. J Dent Res 2015; 94: 28S-36S.

31) Koike T, Ishizaki K, Ogami K, Ueda T, Sakurai K. Influence of anterior palatal coverage on perception and retention in complete dentures. J Prosthet Dent 2011; 105: 272-279.

32) Macura-Karbownik A, Chladek G, Zmudzki J, Kasperski J. Chewing efficiency and occlusal forces in PMMA, acetal and polyamide removable partial denture wearers. Acta Bioeng Biomech 2016; 18: 137-144. 